\title{
State Estimation and Tracking using Fuzzy-Kalman Filter
}

\author{
Raja Gopal Reddy B ${ }^{1}$, Bhanu Prasad C ${ }^{2}$, Padamati Harika ${ }^{3}$, \\ Soma Keerthi Sonam ${ }^{4}$ \\ ${ }^{1}$ Associate Professor, ${ }^{2,3,4}$ Assistant Professor, Vardhaman College of Engineering Hyderabad, India.
}

\begin{abstract}
The aim of this paper is to demonstrate the development of Fuzzy Kalman filet for target tracking problems. The approach is relatively novel. A Comparison with Kalman filter is carried out. The performance both the filters is evaluated and it is found that Fuzzy Kalman filter shows better performance as compared Kalman filter.
\end{abstract}

Keywords: Target tracking, Fuzzy logic, Fuzzy Kalman filter, Kalman filter, Performance evaluation

\section{Introduction}

IT is essential to get accurate information about target states such as position, velocity, and acceleration from the noisy measurements originating from single source or multiple sources. Kalman filter (KF) is a suitable algorithm for such applications. In case of multiple sources, either single KF can be used by fusing the measurements at data level or by state vector fusion (SVF). In case of nonlinear system and measurement models, extended KF (EKF) is used wherein dynamics are linearised with respect to predicted/estimated system states. The accuracy of estimated/fused states depends upon: (i) how accurate the target and measurement models are, and (ii) process noise covariance $Q$ and measurement noise covariance $R$ that basically decide the bandwidth of a filter. In many situations, mathematical models are not known accurately or difficult to obtain. In practice, modelling errors are compensated by tuning the filter, for $Q$, using trial and error or some heuristic approach. A proper combination of fuzzy logic (FL) and KF and a fuzzy Kalman filter (FKF) is investigated for target tracking applications. The performances of KF and FKF are compared for a chosen tracking problem.

\section{Description of Kalman Filter}

The Kalman filter is a technique for estimating the unknown state of a dynamical system with additive noise. The KF has long been regarded as the optimal solution to many tracking and state prediction tasks [1]. The strength of KF algorithm is that it computes on-line. This implies that we don't have to consider all the previous data again to compute the current estimates; we only need to consider the estimates from the previous time step and the current measurement. Popular applications include, state estimation [6], navigation, guidance, radar tracking [2], sonar ranging, satellite orbit computation, etc. These applications can be summarized into various classes such as denoising, tracking and control problems. The basic KF is optimal in the mean square error sense (given certain assumptions), and is the best possible of all filters, if state and measurement inputs are Gaussian vectors and the additive noise is white and has zero mean [1].

We now begin the description of the KF. The block diagram of basic discrete time kalman filter is shown in Figure 1. We assume that the system can be modelled by the state transition equation,

$$
X_{k+1}=A X_{k}+B U_{k}+W_{k}
$$

where $X_{k}$ is the state at time $k, U_{k}$ and $W_{k}$ are an input control vector and additive noise from either the system or the process respectively. $B$ is the input transition matrix and $A$ is the state transition matrix.

The measurement system can be represented by a linear equation of the form,

$$
Z_{k}=H X_{k}+V_{k}
$$

where $Z_{k}$ is the measurement prediction made at time $k, V_{k}$ is additive measurement noise and $H$ is the observation matrix. The KF uses a feed-back control for process estimation. The KF algorithm consists of two steps: $\quad$ a prediction step and an update step as described below.

Prediction (time-update): This predicts the state and process covariance at time $k+1$ dependent on information at time $k$. 
Update (measurement update): This updates the state, process covariance and Kalman gain at time $k+1$ using a combination of the predicted state and the observation at time $k+1$.

Summary of Kalman Filter Equations are given below:

Step1: Predicted Sate

$X_{k+1 / k}=A_{k} X_{k / k}$

Step2: Predicted Measurement

$Z_{k+1 / k}=H_{k} X_{k+1 / k}$

Step3: Predicted Sate Covariance

$P_{k+1 / k}=A_{k} P_{k / k} A_{k}^{T}+Q_{k}$

Step4: Predicted Kalman Gain

$K_{k+1}=P_{k+1 / k} H_{k}^{T}\left[H_{k} P_{k+1 / k} H_{k}^{T}+R_{k}\right]^{-1}$

Step5: Actual Measurement

$Z_{k+1}$

Step6: Updated (Estimated or Corrected) Sate

$X_{k+1 / k+1}=X_{k+1 / k}+K_{k+1}\left(Z_{k+1}-Z_{k+1 / k}\right)$

Step7: Updated (Estimated or Corrected) Sate Covariance

$P_{k+1 / k+1}=\left(1-K_{k+1} H_{k}\right) P_{k+1 / k}$

The above seven equations constitute Kalman Filter Algorithm [1]. By knowing the initial conditions (State X and its Covariance P) and the noise covariance matrices (Process noise Q and Measurement noise R) the steps 1 to 4 can be executed. As soon as measurement is available steps $6 \& 7$ can be executed and cycle can be repeated for next measurement.

\section{Fuzzy Logic Based Kalman Filter}

Fuzzy logic is a multi-value logic used to model any event or condition that is not precisely defined or known. In the FL-based system, one uses: (i) membership function that converts the input/output crisp values to corresponding membership grades indicating its belongingness to respective fuzzy set, (ii) rule base consisting of IF-THEN rules, (iii) fuzzy implications maps the fuzzified input to appropriate fuzzified outputs, (iv) aggregation. to combine the output fuzzy sets (single output fuzzy set for every rule fired) to single fuzzy set, and (v) defuzzification to convert aggregated output fuzzy set from its fuzzified values to equivalent crisp values.

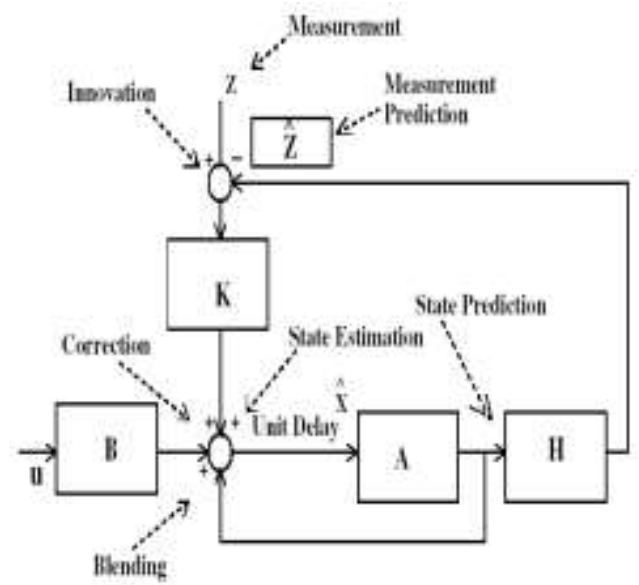

Fig.1 Block Diagram of Kalman Filter

In a KF, since the innovation sequence is the difference between the sensor measurement and the predicted value based on filter's model, this mismatch can be used to perform the required adaptation using fuzzy logic rules4. The advantages derived using the fuzzy techniques are the simplicity of the approach, the possibility of accommodating the heuristic knowledge about the phenomenon, and the relaxation of some of the a priori assumptions of the process. This aspect is accommodated in Eqn (6) as given by 
$X(k+1 / k+1)-X(k+1 / k)=C(k+1)$

where $C(k+1)$ is the fuzzy correlation variable (FCV) and is a nonlinear function of the innovations. To find $C(k+1)$, the innovation vector $e$ is first separated into its $x$ and $y$ components, for 2-D target tracking applications: $e x$ and $e y$. It is assumed that target motion in each axis is independent. The FCV consists of two inputs (i.e., ex and $e y$ ) and single output $\mathrm{C}(k+1)$, where $e x$ is computed by $e x=e x(k+1)-e x(k+T)$ where, $T$ is the sampling time interval in seconds. Interestingly, the FKF can be used to track a manoeuvering or a nonmanoeuvering target. In any fuzzy inference system (FIS), fuzzy implication provides mapping between input and output fuzzy sets. Basically, a fuzzy IF-THEN rule is interpreted as a fuzzy implication. The antecedent membership functions that define the fuzzy values for input ex and ex, are shown in Figs 3 and 4, respectively. Similarly membership functions for output $\mathrm{C} x$ are shown in Fig. 5. The labels used in linguistic variables to define membership functions are LN (large negative), MN (medium negative), SN (small negative), ZE (zero error), SP (small positive), MP (medium positive), and LP (large positive). The rules for the inference in FIS are created based on the past experiences and intuitions. For example, one such rule is: IF (ex is LP) AND ( $\dot{e} x$ is LP) THEN $c x$ is LP (10) This rule is created based on the fact that having $e x$ and $\dot{e} x$ with large positive values indicates an increase in innovation sequence at a faster rate. The future value of $e x$ (and therefore $\dot{e} x$ ) can be reduced by increasing the present value of $c x$ with a large magnitude. Table 1 gives 49 rules5 needed to implement FCV. Output $c x$ at any instant of time can be computed using the input $e x$ and $\dot{e} x$, input membership functions, rules mentioned in Table 1. The properties of FIS used in the present work are: (i) FIS type: mamdani, (ii)AND operator: min, (iii) OR operator: max, (iv) fuzzy implication method: min, (v) aggregation method: max, and (vi) defuzzification method: centroid.

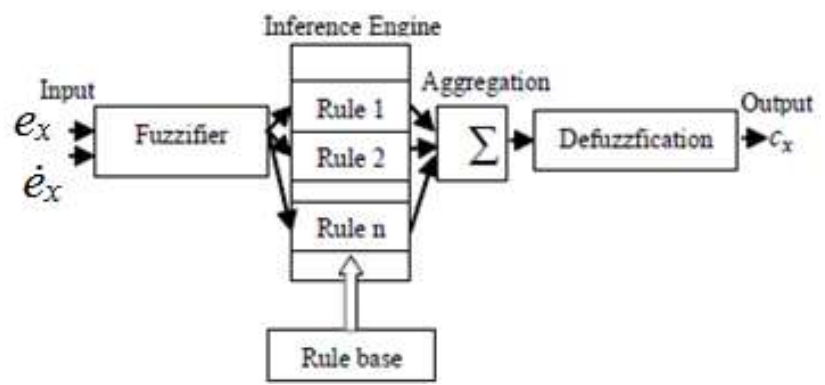

Fig. 2. Schematic of a fuzzy inference system.

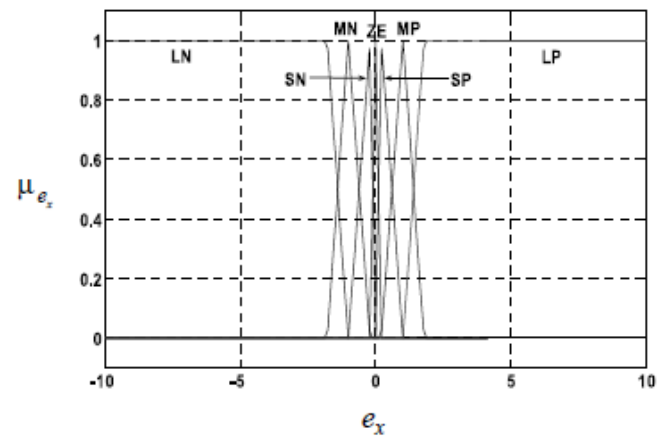

Fig. 3. Membership functions for input ex (indicates degree of membership)

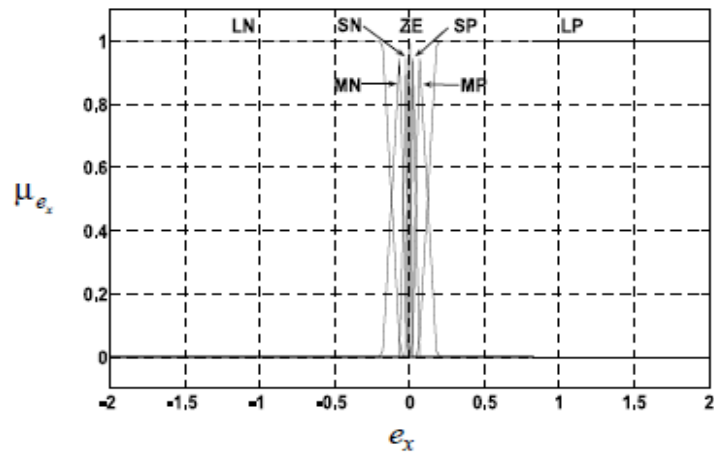

Fig. 4. Membership functions for input $\dot{e x}$ ( $\mathrm{m}$ indicates degree of membership). 


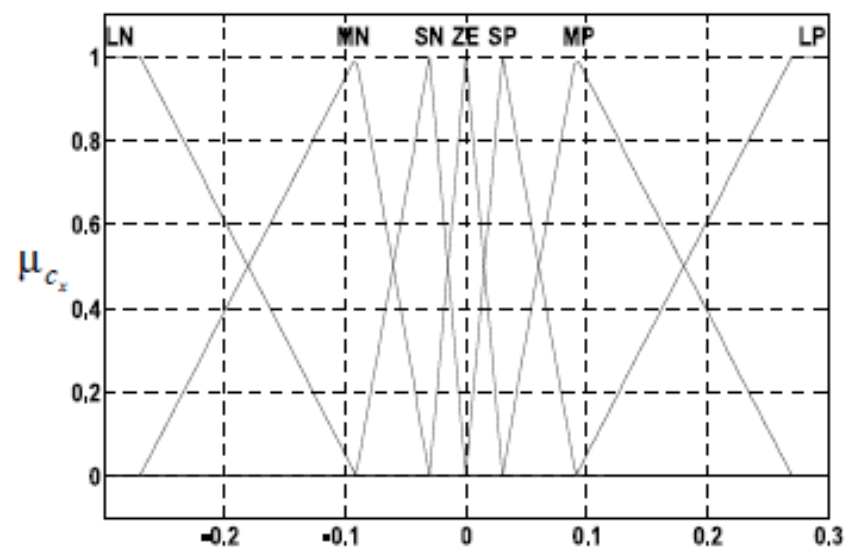

Fig. 5. Membership functions for output $c x$.

\begin{tabular}{|c|c|c|c|c|c|c|c|}
\hline \multirow[t]{2}{*}{$\dot{e}_{x}$} & \multicolumn{7}{|c|}{$e_{x}$} \\
\hline & $L N$ & $M N$ & $S N$ & $Z E$ & $S P$ & $M P$ & $L P$ \\
\hline$L N$ & LN & LN & MN & $\mathrm{MN}$ & $\mathrm{MN}$ & SN & $\mathrm{ZE}$ \\
\hline$M N$ & LN & MN & MN & $\mathrm{MN}$ & SN & $\mathrm{ZE}$ & SP \\
\hline$S N$ & $\mathrm{MN}$ & $\mathrm{MN}$ & $\mathrm{MN}$ & $\mathrm{SN}$ & $\mathrm{ZE}$ & SP & $\mathrm{MP}$ \\
\hline$Z E$ & $\mathrm{MN}$ & $\mathrm{MN}$ & SN & $\mathrm{ZE}$ & SP & MP & $\mathrm{MP}$ \\
\hline$S P$ & $\mathrm{MN}$ & SN & $\mathrm{ZE}$ & SP & MP & MP & $\mathrm{MP}$ \\
\hline$M P$ & SN & $\mathrm{ZE}$ & SP & MP & MP & LP & LP \\
\hline$L P$ & $\mathrm{ZE}$ & SP & MP & MP & MP & LP & LP \\
\hline
\end{tabular}

Table 1. Fuzzy associated memory for output $C x$ with 49 rules

\section{Simulation Experiments}

Simulation studies were performed for (A) state estimation problem [6] and (B) tracking problem. The configuration for the KF and RNN for each of these problems is described below.

\section{Tracking Problem}

Kalman Filter: The state can be described as

$X(t)=[x(t) ; \dot{x}(t)]^{T}$

The state update equation and measurement equation are given by:

$X(t)=A X(t-1)+W(t)$

$Z(t)=H X(t)+V(t)$

where $A=\left[\begin{array}{ll}1 & 1 \\ 0 & 1\end{array}\right], H=\left[\begin{array}{ll}1 & 0 \\ 0 & 1\end{array}\right]$,

$Q_{k}=\left[\begin{array}{cc}1 / 3 & 1 / 2 \\ 1 / 2 & 1\end{array}\right] \sigma_{q}^{2}$ and $R_{k}=\sigma_{r}^{2}$

where $Q_{k}$ and $R_{k}$ are the process and observation noise covariance matrices and $\sigma_{q}^{2}=0.01 ; \sigma_{r}^{2}=0.1$.

\section{Discussion}

Figure 6 depicts the simulation of tracking problem. Figures 6(a) and 6(b) show the performance of KF versus FKF with respect to tracking of position and velocity of a vehicle, respectively. Figures 6(c) and 6(d) depict the corresponding error plots of position and velocity. It is evident from these figures that the difference between the desired and estimated values (tracking error) for FKF are almost zero whereas that with KF is not 
zero but appears to be a random value with zero-mean. The tracking error behaviour of KF is in expected lines as per the algorithm. Kalman filter is a simple, on-line, optimal algorithm but works only for linear systems with Gaussian noise. FKF is expensive in terms of space and time complexities. However, nonlinear approximation can be achieved and there is no restrictive Gaussian assumption with FKF.

\section{Conclusion}

Several variants of fuzzy logic-based Kalman filters are evaluated using simulation data for target tracking. To track manoeuvering target, FCV is re-designed using training and checking data sets obtained from simulated true and measured target positions. It is clear that FKF gives comparatively better performance than KF. It is suggested that just sufficient number of rules could be used rather than a large number of rules to develop an efficient FCV, and in turn, good FKF. KF, and FKF. The performance of EKF and FEKF is compared using for the data generated and it is found that FEKF yields better estimates than EKF, especially in the region where target manoeuvers.

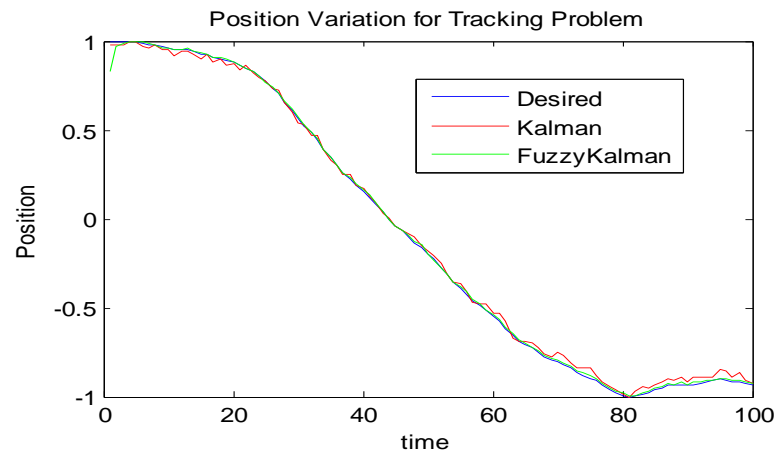

Fig. 6(a)

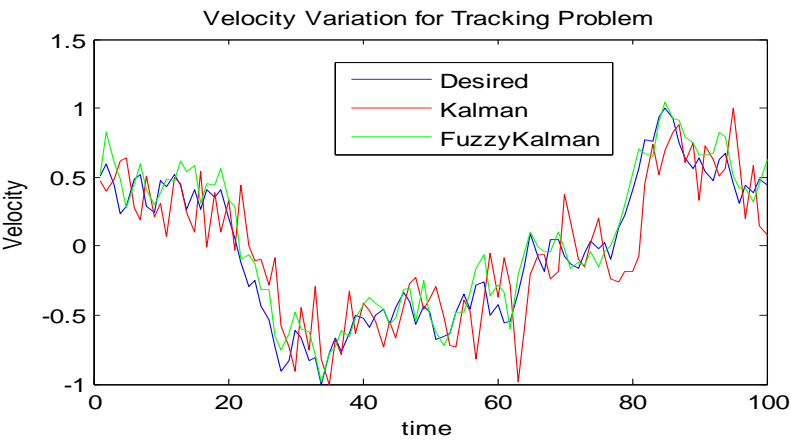

Fig. 6(b)

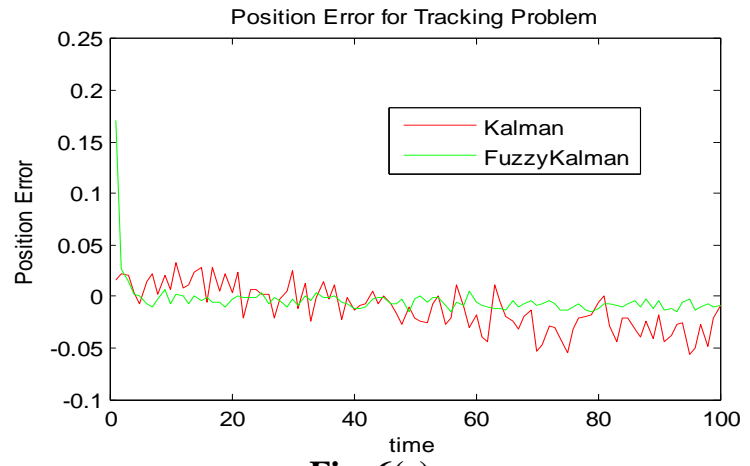

Fig. 6(c) 


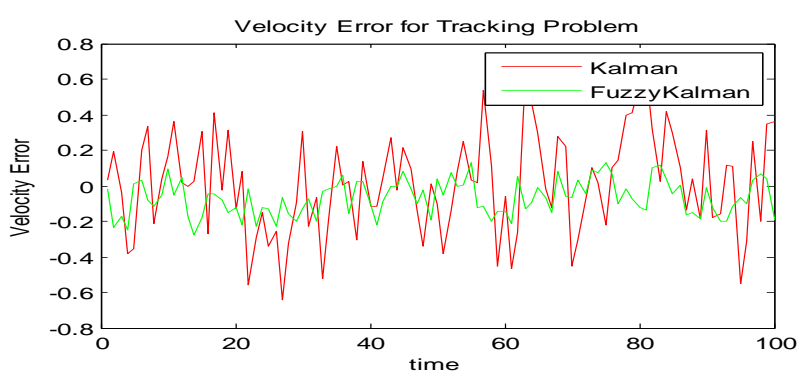

Fig. 6(d)

\section{References}

[1]. Passino, K.M. \& Yurkovich S. Fuzzy control. Addison-Wesley, 1998

[2]. Kashyap, S.K. \& Raol, J.R. Unification and interpretation of fuzzy set operations. In CCECECCGEI, IEEE Canadian Conference on Electrical and Computer Engineering, Ottawa, Canada, May 2006. pp. 353-58.

[3]. Kashyap, S.K. \& Raol, J.R. Interpretation and unification of fuzzy set operations and implications, accepted for publication in Journal of Systems, Science and Engineering (JSSE), Systems Society of India, VSSC, Thiruvananthapuram, India.

[4]. Raol, J.R.; Girija, G. \& Singh, Jatinder. Modelling and parameter estimation of dynamic systems. In IEE Control Engineering Series Book, Vol. 65 IEE, London, August 2004.

[5]. Klein, L.A. Sensor and data fusion: A tool for information assessment and decision making. SPIE Press, Washington, USA, 2004

[6]. David, L. Hall \& Llinas, James. An introduction to multisensor data fusion. Proceedings IEEE, January 1997, 85(1).

[7]. Nirmala, D. Egfin; Vaidehi, V. \& Gandhi, S. Indira. Data fusion using fuzzy logic for multi target tracking. In Proceeding of International Radar Symposium India, 19-22 December 2005. pp. 75-80.

[8]. Peter, S.Maybeck. Stochastic models, estimation, and control, Vol. 2.Academic Press Inc., London Ltd., 1982. 